\title{
Choosing, changing or adhering to a registered doctor in a managed care plan: what will it take? A qualitative survey in rural Mpumalanga, South Africa
}

\author{
a Chabikuli N, MBChB, MCFP(SA), MFamMed ,MSc \\ b Murray M, BA, BA Hon, BD, MDiv, DD \\ ${ }^{c}$ Fehrsen SG, BA, MBChB, MFGP(SA) \\ ${ }^{a}$ Hugo JF, MBChB, MFamMed
}

${ }^{a}$ Department of Family Medicine, Faculty of Health Sciences, University of Pretoria

${ }^{b}$ Faculty of Theology, University of Pretoria

${ }^{c}$ Enablemed (Pty) Ltd

Correspondence to: Prof N Chabikuli, e-mail: nzapf.chabikuli@up.ac.za

Abstract

Background: The phenomenon of doctor shopping has not yet attracted the attention of managers in the South African healthcare sector. Satisfaction with services is known to be mediated by the personal connection inherent in interpersonal continuity with the provider. Such a connection hinges on the fulfilment of expectations of what a good doctor should be at a relational level. With the advent of managed care in South Africa, the restriction on doctor choice and use is being enforced. There is evidence that adherence to one doctor has clinical and financial benefits. Clinical benefits are related to continuity of care, whilst financial gains to the patient and the healthcare system are acquired mainly through better co-ordination of care to avoid inefficiency and wastages. This study explores the concept and context of a 'good doctor', a prerequisite for adherence to one doctor, among members of a health plan targeting the low income community in South Africa.

Methods: This qualitative enquiry of what constitutes a 'good doctor' took place in the form of focus group discussions and key informant interviews and was conducted among members of a managed care health plan in Piet Retief, South Africa. The plan was designed specifically for the employed but uninsured population, a population group of strategic importance in the South African healthcare market. The participants in this study were purposively sampled to include a wide range of opinions about the characteristics of a good doctor.

Results: The respondents looked for values in a doctor that range from fairness, lack of discrimination, autonomy, dignity, warmth and taking time to do a job properly. Generally, the participants expressed willingness to adhere to one registered doctor on condition that the doctor was a 'good doctor'. The definition of a good doctor as provided by the participants was not based on strict technical/clinical criteria, but rather on the patients' and the community's recent experiences of care under that particular doctor. The typical good doctor is a popular, friendly person who does not discriminate along racial lines, listens seriously to anything presented to him or her and examines the patient properly. He/she takes the patient's illness seriously, refers when necessary and gives sick leave that can be used to see a traditional healer. The determinants of the choice of doctor were practical (e.g. the cost), although many were humanistic and difficult to quantify (e.g. word of mouth from fellow patients; the attitude of the doctor toward people; the popularity of the doctor in the community). The specific needs of the respondents relating to traditional healing, migration and sick leave are not readily captured in a standard medical aid contract.

Conclusion: Some characteristics of a 'good doctor' that are particular to the study population include emphasis on a non-discriminative approach, the value of understanding the patient, acceptance of traditional healing and the provision of sick leave when needed. This has important implications for the training of doctors (e.g. to expand the curriculum to include humanistic competencies) and the nature of a managed care contract. 


\section{Introduction}

The phenomenon of shopping for doctors has not yet attracted the attention of managers in the South African healthcare sector. However, there are indications that patients do switch providers, especially from the public to the private sector, in reaction to perceived poor service. ${ }^{1,2}$ When this occurs too frequently, the clinical benefits derived from continuity of care are lost. ${ }^{3}$ From the management point of view, these benefits are mostly related to the informational and longitudinal dimensions of continuity. ${ }^{4}$ There is potential for cost escalation when co-ordination and information flow are disrupted, and also when some para-clinical tests and prescriptions, for example, are duplicated by different doctors for the same condition. With the advent of managed care in South Africa, the restriction on doctor choice and use is being enforced as a possible solution to cost escalation. The member of the scheme is offered a choice of one doctor from a list of accredited providers. Once this choice is made, the member is expected to see that doctor every time. It is not clear, however, what informs a member's choice of doctor. A realisation by the member at a later stage that the doctor initially chosen is not satisfactory may lead to complaints about the restriction and 'doctor shopping'. Little is known of the effect of such restriction, although patient satisfaction is known to be mediated by the personal connection inherent in interpersonal continuity with the doctor. ${ }^{4}$ Satisfaction in this sense is less technical, and more about the fulfilment of expectations of what a good doctor should be at a relational level. The shape of these expectations is not universal. It varies with the nature of the need, and the levels of vulnerability of patients. ${ }^{5}$

Managed care owes its existence to two factors: the runaway escalation of costs and the need to improve on the quality and outcome of healthcare services. In the most basic way, society has come to ask the question: are we getting value for the money invested in healthcare services? Three key role players are involved in the production of health services: the patient and the family (first party), the healthcare provider (second party) and the medical insurance/ medical aid (third party). Managed care seeks to define the roles and responsibilities of each party in such a way that the escalation of healthcare costs is contained and the benefits of the services offered to the patient are optimised. ${ }^{6,7}$ Of the three, the healthcare provider is the best informed on technical matters and is therefore at an advantage when deciding on how much of the resources should be spent on a particular episode of illness (asymmetry of information). Hence managed care interventions tend to be targeted at the provider's behaviour, in the form of financial risk shifting and restrictions in clinical decisions identified as major cost drivers (e.g. hospitalisation, referrals to specialists, prescribing and laboratory investigations).

This study explores the concept of a 'good doctor' that informs the initial choice of a doctor in a managed care scheme. The primary focus of this study was to gain insight into the factors that inform the choice of a doctor by a member of a managed health care plan upon joining the scheme. The study was conducted among members of the Makoti Health Plan, managed by Enablemed, a managed care organisation based in Pretoria, South Africa. This managed care organisation applies restrictions in doctor use to its contracted providers and members. The study was prompted by the realisation that sticking to one healthcare provider is a new 'culture' in the previously unregulated private healthcare market in South Africa. An earlier unpublished report of the Makoti Health Plan suggests that there is a degree of unhappiness with the requirement of sticking to one doctor. ${ }^{8}$

\section{Methodology}

This study attempts to understand, from the perspective of the medical aid member, the meaning attached to the concept of a good doctor that might influence the choice of one provider over the other when seeking care in the relatively new context of managed care in South Africa. Because so little has been published on this topic in South Africa, and on the managed care environment specifically, the study adopted an exploratory, qualitative approach to uncover the problem among members of the Makoti Health Plan and to generate a hypothesis.

The study was conducted in Piet Retief, a rural town in Mpumalanga province. The majority of the members of the Makoti Health Plan in Piet Retief are black Africans from a rural background. The Makoti Health Plan specifically serves the employed but uninsured population, also known as blue collar workers. Most of the members are employed in the timber industry. Being migratory and family dislocation are common features of blue collar workers. Employment benefits, including healthcare insurance, are usually negotiated between management and the unions on behalf of the workers. But the choice of a particular doctor within the chosen plan is the worker's responsibility, presumably in consultation with the spouse.

Participants were purposefully identified from the Makoti Health Plan membership register. The purpose of the sampling was to include a wide range of opinions on the characteristics of a good doctor from a variety of sections of the study population, including factory managers, labour union representatives, main members of the medical aid and their immediate families. Confidentiality was assured in letters of invitation which were explained by the researcher. Confirmation of participation was followed up telephonically.

Since illness and health-seeking choices are very personal issues, where differences in preferences exist even within families, seven focus group discussions (FGD) were conducted with the main members of the plan and their families. This technique is useful and widely used to unearth a range of views among participants with similar experiences. Groups were created by gender and age to make them homogeneous. The exploratory question, "how do you recognise a good doctor?' was asked to start the discussion in the focus group. There were not enough managers and union representatives to form groups, hence eight individual interviews were administered using the same exploratory question.

All the interviews were conducted in Piet Retief, outside the work premises and after working hours between 15 August and 22 September 2006. Two teams facilitated the interviews using audiotapes to capture data until saturation was reached. The teams were made up of two consultants (first two authors) and three senior Makoti Health Plan staff with academic background and extensive research experience. Harmonisation meetings and training were held during the planning and implementation phases. The same team that collected the data was involved in the analysis. Analytical categories were used to describe the participants' constructs of a good doctor. These categories were obtained gradually as they emerged from the researcher's interaction with the data. Members of the research team listened to the tapes, and read and re-read the transcribed interview materials to immerse themselves in the data and identify and index as many categories as possible. These emerging categories were shared during the harmonisation meetings. As such, the study benefited from more than one analyst, giving it greater consistency and reliability. 
The data were then organised into a reduced number of themes by the 'cut and paste' technique. The themes were interpreted and their associations identified to fit the description of a good doctor.

Ethics approval to conduct this study was obtained from the University of Pretoria.

\section{Findings}

\section{Attributes of a good doctor}

The respondents seemed to look for certain values over and above the treatment and cure that the healthcare provider may offer. These values ranged from fairness, lack of discrimination, autonomy, dignity, warmth and taking time to do a job properly.

\section{It emerges that the following characterise a good doctor:}

- Many patients come to see him or her

- Friendly and smiles

- Chats with me when we meet in the street

- Treats people the same and does not discriminate

- You can present everything to him or her without fear

- Listens to you; provides a quality service even when he/she has many clients

- Examines you properly and takes time when doing so; shows exactly what's happening

- Gives good medication and adapts treatment if necessary; gives injections

- Is serious about my illness, gives sick leave

- Sits down and talks to me before he/she gives medication

- If cannot help, he/she can tell me of someone else that could help me

- Gives days off to go to the traditional healer

The absence of these attributes in various combinations prompts members to change providers. The deficit of some attributes can be compensated for by the strength of other attributes. This creates a degree of tolerance: if the doctor does not immediately live up to expectations, he might get another chance to improve. One respondent reported that "you can go back for a second time, but not for a third time". Some respondents, for example, reported experiencing what they perceived to be racial discrimination from a particular practice, but they still continued to consult the doctor, attracted by other good reasons or attributes.

\section{Willingness and barriers to adhere to a doctor}

Generally, the participants expressed willingness to adhere to one registered doctor, on condition that the doctor was a 'good doctor'. The definition of a good doctor as provided by the participants is not based on strict technical/clinical criteria, but rather on the patients' and the community's recent experience of care under that particular doctor. It is important to note that the term 'doctor' refers to 'Western doctors' distinct from the 'traditional healers', whose competence seems to be judged by a different frame. The participants' explanations of the process that leads to the choice of and satisfaction with a doctor suggest a certain degree of consumer activism, even though not totally informed on the technical clinical competencies. Some determinants for the choice of a doctor are practical (e.g. "the cost involved for me or my family to travel to that practice"), but many more are subjective or humanistic and difficult to quantify (e.g. word of mouth from fellow patients; the attitude of the doctor toward people; the popularity of the doctor in the community).
The following are among conditions that could definitely result in a doctor's practice being deserted:

- If you experience persistent problems and the treatment is not good

- If the doctor is too busy or running another business

- Shifting the responsibility to student doctors

- Discrimination: when doctors discriminate against patients on the basis of race it impacts negatively on the patient's healthcare experience. One respondent relates the following experience: "Sometimes you arrive, a white person enters they just say to you: wait, whereas you have been in a queue for a long time" (Team 1: Intvw1).

- Not getting sick leave: conflicts tend to arise from differences between the doctor and patient in judging how ill or fit a patient is. Sometimes, patients are dissatisfied with doctors because of unrealistic expectations: "If he goes to the doctor he must at least be off for two days whether it is a headache or a painful eye but he must be off" (Team 2: Intvw3).

The personal circumstances of migrant workers require a choice of more than one doctor to attend to the main member of the Health Plan and his/her family left behind in the village/town of origin. A strict interpretation of the restriction (i.e. one doctor per member and family) has caused much unhappiness among the participants. Many members still believe the choice of doctor is very rigid: "(...) never allow us to go to other doctors. Sometime you wish to change, but that's not possible" (Team 1: Intvw2). The cost of this misinterpretation of benefits to the medical aid member is important. It relates to a loss of time, energy and transport fare or even paying cash out of pocket to the unregistered doctor. "If I'm at home and I get sick there ... I have to travel all the way from home up until here, whereas there are some doctors that side and we don't have transport and I am sick and there is a doctor nearby" (Team 2: Intvw4). The family structure and dynamics amongst migratory labourers could have an influence on their experience of their healthcare benefits. For example, having a wife and a girlfriend with babies in different towns is common, but "she never consulted because with this card... you can't consult if you're in Durban and your doctor is in Piet Retief". (Team 1: Intvw2). Transport is very expensive for these workers and takes up a substantial part of the household budget.

The findings of this study also suggest an informal classification of the various causes of the illness into those that need Western and others that need traditional healthcare solutions. Visiting traditional healers was a prevalent theme among the respondents, with a clear modus operandi. "Most of the time when they want to see a traditional healer they go through the Western doctor first so that they can get a medical certificate and then if they have that then they go to the traditional healers" (Team 2: Invtw3).

Similarly, the unwritten scope of practice defines whether the traditional healer must be consulted instead of the doctor. A doctor might be a good doctor and earn respect but might not be right to cure a specific illness or disease. One respondent explained that "like an African... if you see some things happening, you must know that you must go to traditional healer...Knowing that Western medicines can't do nothing... In that way I'm forced to go to a traditional healer...I know a Western doctor won't be able to treat me the way it suppose to... maybe you're sick as a results of your ancestors...In this case you'll be forced to go 
to a traditional healer...Western doctors knows nothing about this" (Team 1: Intvw5). Visits to traditional healers are still prevalent and fall outside the medical aid restrictions. The hypothesis regarding the interpretation of illness as a type of power that befalls someone was also reported in the 2005 study. In the near future, however, the traditional health sector will be formalised and it will be necessary for medical aids to consider contractual arrangements with traditional health practitioners.

The lack of objectivity in this definition of competence is bound to create challenges in the requirement to stick to one doctor, as no one provider can be good to all at all times. As one clinic sister reflects: "They can't...they can't... it's not in them. We talked about this before; they can't stick to one doctor... They can stay with that doctor for some time but at some stage he will say no... he couldn't heal my child with this ...now l'll take this one... Or he will hear somebody talking about the other one, then he will change to the other doctor. I don't know why, but they can't stick to one doctor for a long time (Team 2: Intvw3).

\section{Discussion}

The use of a different data collection tool for managers and union representatives is in principle a limiting factor. However, the researchers administered the same question in individual interviews and focus group discussion, and the harmonisation meetings looked specifically at any trends that might suggest that there were biases between the two groups. The attributes of a good doctor described in this study are similar to those described in other contexts as concrete examples of professionalism that appeal to patients. ${ }^{9}$ These include features such as greeting, privacy and proper physical examination, which are also valued in the black African culture in South Africa. Another study, using a discrete choice experiment, has suggested that similar attributes (doctor listens, easily understood information, shared treatment decision, more information and longer consultation) are "utility bearing" and highly valued by patients. ${ }^{10}$

The overall evaluation of the goodness of doctors is a flexible balancing act including past experiences and the current social standing of the doctor's name, and is often re-evaluated over time. In the study community, however, it seems as if the humanistic attributes (include communication and relationships) are very important because of ignorance of the clinical or technical attributes that can only be judged by the outcome: after all is done, did I get better? A similar situation has also been described in settings as different from South Africa as Japan. ${ }^{11}$

In a way, perceptions drive satisfaction. As these perceptions change with specific incidents, so will satisfaction. Satisfaction is therefore not a permanent feature in clinical settings and needs to be reevaluated regularly. This has implications for adherence to a disease management plan for chronic conditions, especially those with stigma such as HIV and AIDS.

Some features, however, are specific to the context of our study population. Things like linkages to traditional healing, discrimination, especially when racial in nature, and the shared, communal experience of the doctor are specific to the black South African context. They are rooted in the culture and the painful discriminatory social experiences of the apartheid system and are still sensitive issues in all spheres of social functioning. ${ }^{12,13,14} \mathrm{~A}$ service and curriculum that do not sensitise healthcare workers to these social issues are not appropriate for the
South African setting.

The doctor liked for his/her willingness to book the patient off sick any time the request is made is interesting. It is important to note that most patients in this community engage in demanding manual labour that can exert a toll on the physical condition of the individual, who then may require rest. This may be completely unlinked to the severity of the clinical problem as presented. Also, the distance from family may exert pressure to be away from work from time to time for social reasons. A doctor that understands these needs is most likely to be rated highly.

Word of mouth is a widely used means of sharing experiences of treatment received from a particular GP. The power of word-of-mouth communication should not be underestimated in a society where oral tradition has long been part of the way of life. Suggestion boxes have performed poorly as a means to get patients' opinions of services rendered in many public healthcare facilities in South Africa. Typically, patients vote with their feet and share their experiences with relatives and friends, who will use this in their own decisions about healthseeking behaviour.

How does one influence the way in which people evaluate their own illnesses and their healer? Some of the attributes of the doctor that have been described can be taught and measured (e.g. professionalism). The more subjective attributes require only heightened awareness and willingness to negotiate within the bounds of professional ethical and legal limits. The bond that exists between traditional healer and client, for example, is almost on a subconscious/ intuitive level. "I am his patient (but) he charge me smaller amount because I am (like) his son and he is like my father (Union:Resp1\&2).

\section{Conclusions}

There are similarities between the defining characteristics of a good doctor in the studied community and what has been described elsewhere. These characteristics relate to professionalism and humanism. Although individual characteristics are clearly spelled out and defined, the overall assessment of the standing of the service provider is a complex balancing act of good and bad characteristics. Often, the end result is taken into consideration when deciding to change doctors. Even then, the decision is not taken in haste. The provider is given 'a second chance'.

Some characteristics particular to the study population include emphasis on discrimination on the basis of race, the value of understanding and linking with traditional healers, and the importance of booking patients off duty when requested. These issues are firmly rooted in the socio-cultural dimension of the member's functioning and are rarely taken into consideration by the prevailing biomedical paradigm.

Once the standing of the service provider has been established, whether positive or negative, this information is shared within the community by word of mouth. Rumours are an important and trusted source of information about the qualities and competency of a healthcare provider in this community.

Healthcare providers and managers need to be aware of and respond to these issues in order to provide a service valued by patients and their families.

\section{Acknowledgements}

This paper draws from a research project that was funded by 
Enablemed (Pty) Ltd. Nzapfurundi Chabikuli co-supervised the design, reporting and analysis of the work with the second author. He jointly had the idea for the article with the other three authors, performed the literature search and wrote the first draft of the article. As such, he is the guarantor of the paper.

\section{References}

1. Central Statistical Services. South Africa General Household Survey, 2005. Pretoria: Central Statistical Services.

2. Volmink J, Laubscher J, Furman S. The SASPREN primary care survey - who consults the family doctor? S Afr Med J 1996;86(3):241-5.

3. Donahue KE, Ashkin E, Pathman DE. Length of patient-physician relationship and patient's satisfaction and preventive service use in the rural south: a cross-sectional telephone study. BMC Fam Prac 2005(6):40.

4. Saultz JW. Defining and measuring interpersonal continuity of care. Ann Fam Med 2003:1:134-43.

5. Nutting PA, Goodwin MA, Flocke SA, Zyzanski SJ, Stange KC. Continuity of primary care: to whom does it matter and when? Ann Fam Med 2003:1:149-55.
6. Takemura Y, Beck JR. Laboratory testing under managed care dominance in the USA. $J$ Clin Pathol 2001;54(2):89-95.

7. Bodenheimer T. High and rising health care costs. Part 3 : the role of health care providers. Ann Intern Med 2005;142(12 Pt1):996-1002.

8. Murray M. Makoti Health Plan as managed by Enablemed Contentment Investigation, June-August 2005. Unpublished research report.

9. Davis RL, Wiggins MN, Mercado CC, O'Sullivan PS. Defining the core competency of professionalism based on patient perception. Clin Experiment Ophthalmol 2007;35(1): $51-4$

10. Longo MF, Cohen DR, Hood K, et al. Involving patients in primary care consultations: assessing preferences using discrete choice experiments. Br J Gen Pract 2006;56(522):35-42.

11. Hagihara A, Tarumi K, Odamaki M, Nobutomo K. A signal detection approach to patient-doctor communication and doctor-shopping behaviour among Japanese patients. J Eval Clin Pract 2005;11(6):556-67.

12. Pinkoane MG, Greeff M, Williams MJ. The patient relationship and therapeutic technique of South Sotho traditional healer. Curationis 2005;28(4):20-30.

13. Puckree T, Mkhize M, Mqobozi Z, Lin J. African traditional healers: what health care practitioners need to know. Int J Rehabil Res 2002;25(4):247-51.

14. Nightingale EO, Hannibal K, Geiger HJ, Hartmann L, Lawrence R, Spurlock J. Apartheid medicine. Health and human rights in South Africa. JAMA 1990;264(16): 2097-102. 